\title{
R. Holzbach \\ Der Benzodiazepinentzug und dessen Behandlung
}

Withdrawal from Benzodiazepines and Treatment Aspects

\section{Zusammenfassung}

Viele Ärzte sehen in der Langzeitverschreibung der Benzodiazepine nur dann ein Problem, wenn die Dosis von den Patienten selber, ohne Absprache mit dem Arzt, gesteigert wird oder die tägliche Dosis zu hoch geworden ist. Die typischen Folgeerscheinungen, die bei einer Langzeitverschreibung auftreten, sind nicht bekannt, werden deshalb auch nicht erkannt und sind somit auch nicht Anlass für ein Ausschleichen der Medikation. Häufig besteht die Vorstellung, dass den Betroffenen der Entzug nicht zugemutet werden könnte. Werden die pharmakologischen Besonderheiten der Benzodiazepine beachtet und der Entzug sowohl von der pharmakologischen Seite als auch der psychotherapeutischen Seite fachgerecht unterstützt, können sowohl komorbide als auch ältere Benzodiazepinabhängige schrittweise von den Benzodiazepinen entzogen werden.

Schliuisselwörter

Benzodiazepine $\cdot$ Entzug

\section{Abstract}

Many physicians consider long-term prescription of benzodiazepines only as a problem, if patients themselves increase their dose without prior consultation with the doctor or if the daily dose became too high. The typical after effects of long-term prescription are not known; they are, therefore, not recognised and do not call for tapering off the medication. The idea frequently prevails that patients would be overtaxed by withdrawal. If the specific pharmacological properties of benzodiazepines are taken into account and if withdrawal is assisted both by pharmacological and psychotherapeutic experts, it is possible to withdraw step by step both comorbid and older benzodiazepine addicts.

Key words

Benzodiazepines · withdrawal

\section{Einleitung}

Langzeitkonsumenten von Benzodiazepinen (BZD), ob im Sinne der Niedrig- oder Hochdosisabhängigkeit, tauchen nur selten in spezialisierten Einrichtungen auf, aber sind wie andere Suchtkranke in allen Bereichen medizinischer Versorgung anzutreffen. Egal ob in haus- oder fachärztlichen Praxen, Akutkrankenhäusern oder in Rehakliniken. Allerdings wird nur selten die Diagnose gestellt [1 -2]. Dies hat sicherlich mehrere Gründe:
1. Falsche Erwartung an die Langzeitwirksamkeit der Benzodiazepine

2. Niedrigdosisabhängigkeit wird nicht als Abhängigkeit gesehen

3. Folgen des Langzeitkonsums (auch niedriger Mengen) sind nicht bekannt/werden nicht erkannt

4. Vorgehensweise bei der Entzugsbehandlung ist nicht bekannt

5. Belastung des Entzuges wird überschätzt

6. Vorteile der Abstinenz werden unterschätzt

7. Unsicherheit wegen hoher psychiatrischer Komorbidität
Institutsangaben

Korrespondenzadresse

Dr. R. Holzbach · Westfälische Kliniken Warstein und Lippstadt · Franz-Hegemann-Str. $23 \cdot 59581$ Warstein · E-mail: ruediger.holzbach@wkp-lwl.org

Bibliografie

Suchttherapie 2006; 7: 97 - 106 @ Georg Thieme Verlag KG Stuttgart · New York

DOI 10.1055/s-2006-927015

ISSN 1439-9903 
Alle Aussagen, die in diesem Artikel zu den BZD getroffen werden, treffen im Wesentlichen genauso auf die so genannten Non-Benzodiazepine Zolpidem, Zopiclon und Zaleplon. Diese gegören chemisch zwar nicht zur Gruppe der BZD dazu, im Gehirn wirksam sie aber über die gleichen GABAergen Rezeptoren (s. unten) und führen genauso zu einer Abhängigkeit (allerdings liegen für Zaleplon bisher keine entsprechenden Kasuistiken vor - der Hersteller hat, im Gegensatz zu den [primären] Herstellern der zwei anderen Substanzen, mit Markteinführung einen entsprechenden Warnhinweis auf das Risiko der Abhängigkeitsentwicklung gegeben).

\section{Zu 1.: Langzeitwirksamkeit}

Viele Ärzte sehen eine Langzeitverschreibung dadurch gerechtfertigt, dass die Patienten die Dosis nicht steigern (s. Pkt. 2) und die Patienten von der Weiterverschreibung profitieren. Defakto gibt es aber keine Indikation für eine Langzeitverschreibung von BZD.

\section{Angststörungen}

Unter der Einnahme nimmt im Verlauf die Angst zu (Wirkumkehr), was zunächst im Einzelfall als Verschlechterung der Angststörung verstanden werden könnte - und ggf. zu einer Dosissteigerung führt. Lemoine [3] konnte aber an einer Gruppe Lorazepamabhängigen mit einer primären generalisierter Angststörung zeigen, dass unter dem BZD mehr Angstzustände auftraten als nach abgeschlossenem Entzug.

Für Patienten mit chronischen Angsterkrankungen werden BZD, gemäß Leitlinie Angsterkrankungen [4], als Langzeitbehandlung der Panikstörung (in der Kombination mit kognitiver Therapie) empfohlen, wenn BZD nur beim Auftreten von Panikattacken genommen werden. Bei der sozialen Phobie und der generalisierten Angststörung wird eher abgeraten (Leitlinie Angsterkrankungen). Bei einer generalisierten Angststörung wird ebenfalls von der Langzeitverordnung von BZD abgeraten [5].

\section{Schlafstörungen}

Bei primären Schlafstörungen (ohne erkennbare Ursache) ist eine Wirkung der BZD für Zeiträume bis zu 4 Wochen belegt, darüber hinaus aber nicht [6-7]. Einschlägige Empfehlungen von Experten(-gruppen)/Leitlinien zum Einsatz von BZD-Hypnotika oder Non-Benzodiazepinhypnotika (Zolpidem, Zopliclon, Zaleplon) beziehen sich ebenfalls auf eine Kurzzeitverordnung [7].

\section{Depressionen}

Auch bei depressiven Störungen gibt es in den einschlägigen Leitlinien die Empfehlung einer initialen Kombinationsbehandlung mit BZD, die aber auf eine Dauer von 2 bis 4 Wochen begrenzt bleiben sollte [8-9]. Auch hier gilt, dass die Symptome des Langzeitkonsums von BZD (s. Pkt. 3) als Verschlechterung der Depression fehlinterpretiert werden können und die Verschreibung, ggf. mit erhöhter Dosis, fortgeführt wird.

\section{Zu 2.: Niedrigdosisabhängigkeit ist keine Abhängigkeit?}

Die Niedrigdosisabhängigkeit ist definiert als eine Langzeitverschreibung von BZD mit einer Dosis bis $20 \mathrm{mg}$ Diazepamäquivalenzdosis. Formal betrachtet erfüllen tatsächlich die wenigsten Patienten in dieser Gruppe die ICD-10 Kriterien für eine Abhängigkeit. Wird diese Niedrigdosisabhängigkeit aber als eine „Substitutionsbehandlung“" verstanden [vgl. 9-10], wird schnell deutlich, warum auch bei dieser Patientengruppe die typischen Symptome des Langzeitkonsums von BZD (vgl. Pkt. 3) auftreten und, dass häufig Patienten dieser Gruppe, sobald sich die Möglichkeit ergibt, rasch die Dosis steigern.

\section{Zu 3.: Folgen des BZD-Langzeitkonsums}

Es erscheint manchmal eher eine akademische Frage zu sein, ob eine Abhängigkeit vorliegt oder nicht. Die Abhängigkeit als solche ist ja nicht ein Leiden, sondern die Folgen der Abhängigkeit. Deshalb ist für die Betroffenen die Frage auch wichtiger, welche negativen Folgen hat der Langzeitkonsum von BZD für mich? Diese treten unabhängig davon auf, ob eine Niedrig- oder Hochdosisabhängigkeit vorliegt. Pauschal kann gesagt werden, dass je länger die Einnahme erfolgt, je höher die (Lebenszeit-)Dosis ist, um so ausgeprägter sind die Symptome. Ein Überblick gibt Tab. 1.

\section{Tab. 1 Symptome des Benzodiazepinlangzeitkonsumes}

führende Trias:
- Gefühlserleben abgeschwächt
- gestörtes Körpergefühl/verminderte körperliche Energie
- Vergesslichkeit und geistige Leistungsminderung
akzessorische Symptome:
- Fähigkeit zur Selbstwahrnehmung/-kritik abgeschwächt
(dadurch werden die Veränderungen nicht/nur eingeschränkt realisiert)
- gereizte Verstimmungszustände
- Überforderung in bzw. Vermeidung von neuen oder belastenden
Situationen
- muskuläre Schwäche, ggf. mit Reflexverlust
- Appetitlosigkeit
- Vermeidung des Themas Tabletten/heimliche Einnahme
- Konfliktvermeidung

\section{$\mathrm{Zu}$ 4.: Vorgehensweise Entzugsbehandlung nicht bekannt}

Diese wird im nachfolgenden Teil des Beitrages ausführlich vorgestellt.

\section{Zu 5.: Belastung des Entzuges überschätzt}

Gerade bei älteren Patienten wird häufig von Kollegen die Meinung vertreten, die Strapazen des Entzuges „lohnen“ sich nicht mehr. Diese Ansicht trifft man häufig bei Kollegen auf Fortbildungsveranstaltungen an, die bisher keine oder keine große Erfahrung mit BZD-Entzügen haben. Eigene Erfahrungen und die von anderen erfahrenen Kollegen zeigen, dass für jeden Patienten ein angemessener Rahmen und ein angemessenes Reduktionstempo gefunden werden kann, wenn der Betroffene das Absetzen der BZD wirklich will und das Reduktionsregime ausreichend flexibel ist. Häufig wird der Punkt der „Zumutbarkeit des Entzuges" mit den dahinter stehenden psychischen Problemen und der Erwartung deren Behandelbarkeit vermischt (s. Pkt. 7).

Auch im Alter ist ein BZD-Entzug nicht riskanter als bei Jüngeren, sofern das Herunterdosieren langsam erfolgt [11].

\section{Zu 6.: Vorteile der Abstinenz unterschätzt}

Dieser Punkt ist eng verknüpft mit den Punkten 3 und 7 - dem fehlenden Wissen um die Folgen des BZD-Langzeitkonsums und der 
Behandelbarkeit der Komorbidität. Es besteht die Erwartung, dass die aktuellen Beschwerden und frühere Symptome nach einem Entzug den Betroffenen ohne Abschirmung durch die BZD treffen würden. Darüber kann aber immer erst nach dem Absetzen der BZD und dem Abklingen möglicher Entzugserscheinungen geurteilt werden und wenn entsprechende andere Behandlungsmethoden greifen (s. Pkt. 7). Entscheidend erscheint hierbei die eigene Erwartungshaltung (des Behandlers) und die daraus resultierende Patientenführung. Zunächst ist es notwendig, den Patienten umfassend über die Problematik der BZD-Langzeiteinnahme zu informieren. Dazu können neben dem persönlichen Gespräch auch entsprechende Broschüren dienen (z. B. von der DHS, Hamm: „Immer mit der Ruhe ...“). Neben der Information über die Entzugserscheinungen und die (regional) möglichen Rahmenbedingungen im Entzug wird es dann im Wesentlichen darum gehen, dem Patienten alternative (aktive) Bewältigungsstrategien für seine Beschwerden aufzuzeigen, damit eine möglichst hohe Lebensqualität erzielt werden kann. Das Fallbeispiel soll dies exemplarisch zeigen.

\section{Fallbeispiel Jürgen S.}

Jürgen S. war bereits als Kind psychisch auffällig, sodass er wegen seiner starken Unruhe von einem Arzt zeitweise Benzodiazepine verschrieben bekam. Nach Schulabschluss und Tontechnikerausbildung musste er beruflich viel unterwegs sein. Dies war ihm stets unangenehm und bereitete ihm diffuse Ängste. Mit 25 Jahren erlitt er in einer fremden Stadt eine erste Panikattacke und erhielt vom Notarzt Diazepam gespritzt. Aufgrund des positiven Effektes ließ er sich zu Hause Diazepam weiter verschreiben. Ärzte, die ihm die Verschreibung verweigerten und vor der Abhängigkeit warnten, mied er. Sein Gedanke - „Ich brauche das Medikament und ich bin niemand, der süchtig wird“ - wurde von einem Arzt geteilt, der ihm deshalb die BZD über Jahre weiter verschrieb - im Wechsel auf Kassen- und Privatrezept. Die Dosis steigerte sich langsam über die Jahre. Mit Ende Vierzig nahm Herr S. täglich 6 Tabletten Bromazepam à $6 \mathrm{mg}$ ein. Er verließ sein zu Hause nur noch zur Arbeit, litt unter diversen Ängsten, überprüfte ständig seinen gehorteten Medikamentenvorrat von rund 1000 Tabletten und ging, wenn es sich gar nicht vermeiden ließ, auf Dienstreisen nur mit „einem Koffer voller Benzodiazepine“. Schließlich war es seine Partnerin, die auf eine Veränderung drängte und Herr S. entschloss sich zu einem Entzug. Dieser verlief für ihn zunächst sehr schwierig in einer anderen Klinik. Im Verlauf wurde er in unserer Ambulanz vorgestellt. Die Gabe von Carbamazepin, die Information über die Entzugserscheinungen und der Kontakt mit anderen Betroffen halfen ihm dann, den Entzug zu bewältigen. Mit Abklingen der Entzugssymptome bekam er zunehmend Zugang zu seinen Gefühlen und Wünschen. Er erlebte banale Alltagssituation sehr intensiv und überwältigend - z.B. blühende Pflanzen verbunden mit großen Glücksgefühlen, aber auch starken negativen Gefühlen (z.B. Fernsehberichterstattung über Terroranschläge). Eine anschließende Verhaltenstherapie, unterstützt durch eine SSRI-Medikation, vermittelte ihm geeignete Strategien im Umgang mit seinen Ängsten, aber auch mit den außerdem bestehen Störungen Kleptomanie, Trichotilomanie und recurrent brief depression (kurze rezidivierende depressive Phasen). Zunehmend erweiterte Herr S. seinen Aktionsradi- us, wurde aktiver, unternahm alleine Bahn- und Flugreisen, kaufte sich ein Auto und verfolgte zielstrebig persönliche Ziele. Er fühlt sich wie neu geboren und trotz krisenhafter Rückschläge (er musste noch einmal stationärpsychiatrisch wegen seiner Ängste behandelt werden), durch die BZD um Jahre seines Lebens beraubt. Er hat deshalb seinen verschreibenden Arzt angezeigt und durch die Schiedsstelle der Ärztekammer einen finanziellen Schadensersatz zugesprochen bekommen.

\section{Zu 7.: Unsicherheit psychiatrische Komorbidität}

Viele Ärzte haben sich nur während ihres Studiums mit psychiatrisch psychotherapeutischen Themen auseinander gesetzt. Dies bedeutet, ohne den Besuch von Vorlesung (freiwillig und wenig besucht), in der Regel etwa 36 Stunden (Pflicht-)Praktikum - für das gesamte Stoffgebiet der Psychiatrie; die meisten Fortbildungen z.B. zur Sonographie oder EKG-Diagnostik, die Ärzte beispielsweise im Rahmen einer internistischen Fortbildung durchlaufen, umfassen mehr Stunden. Dementsprechend ist das Wissen um und Vertrauen in psychiatrisch-psychotherapeutische Methoden nicht besonders ausgeprägt. Egal, ob moderne Psychopharmakatherapie (zumeist auch teuer) oder Psychotherapie (Wartezeit von 6 Monaten und länger) - diese Methoden brauchen Zeit, bis eine Wirkung zu erkennen ist - BZD dagegen helfen schnell. Dies entspricht auch der Erwartung vieler Patienten, die vom Arzt, ohne eigenes großes Zutun, rasche Hilfe bei psychischen Beschwerden erwarten. Kommt nun noch zu der psychischen Grundproblematik die BZD-Abhängigkeit hinzu, erscheint die Situation noch weniger lösbar und die fortgesetzte Verschreibung, solange die Patienten die BZD nicht in hoher Dosis einnehmen, das kleinere Problem.

\section{Pharmakologie der Benzodiazepine}

Um BZD fachgerecht auszuschleichen, bedarf es genauerer Kenntnisse über die Pharmakologie der BZD, insbesondere der Wirkstärke (Äquivalenzdosis) und der Halbwertszeit der verschiedenen Präparate.

\section{Wirkstärke (Äquivalenzdosis)}

BZD sind eine Gruppe von Wirkstoffen, die chemisch untereinander verwandt sind. Bislang wurden mehrere tausend verschiedene BZD synthetisiert [12], wobei aktuell lediglich 23 verschiedene BZD-Wirkstoffe in der Bundesrepublik Deutschland auf dem Markt sind. BZD verstärken die hemmende Wirkung des Neurotransmitters GABA auf die Fortleitung von Nervenreizen. Dadurch führen sie zu einer Entspannung der Muskulatur, sind angstlösend und unruhedämpfend, bis hin zur Schlafinduktion sowie antikonvulsiv wirksam. Es gibt verschiedene Unterformen des GABA-Rezeptors, zu denen die verschiedenen BZD eine unterschiedliche Affinität besitzen, worüber sich gewisse Wirkunterschiede theoretisch ableiten lassen. Auch wenn die verschiedenen BZD dementsprechend unter unterschiedlichen Indikationen (Antikonvulsivum, Anxiolytikum, Hypnotikum, Muskelrelaxanz) auf dem Markt eingeführt wurden, kann im klinischen Alltag trotz der unterschiedlichen Rezeptoraffinität bei äquivalenter Dosierung von vergleichbarer klinischer Wirkung ausgegangen werden (s. Tab. 2). 
Tab. 2 Äquivalenztabelle

\begin{tabular}{|c|c|c|}
\hline Wirkstoff & Handelsname (z. B.) & mg Äquivalenzdosis ${ }^{1}$ \\
\hline Alprazolam & Tafil & 1,5 \\
\hline Bromazepam & Lexotanil & 6 \\
\hline Brotizolam & Lendormin & 0,5 \\
\hline Chlordiazepoxid & Librium & 20 \\
\hline Clobazam & Frisium & 20 \\
\hline Clonazepam & Rivotril & 2 \\
\hline Clotiazepam & Trecalmo & 5 \\
\hline Diazepam & Valium & 10 \\
\hline Dikaliumclorazepat & Tranxilium & 20 \\
\hline Flunitrazepam & Rohypnol & 0,75 \\
\hline Flurazepam & Dalmadorm & 30 \\
\hline Loprazolam & Sonin & 1,5 \\
\hline Lorazepam & Tavor & 2 \\
\hline Lormetazepam & Noctamid & 1,5 \\
\hline Medazepam & Rudotel & 20 \\
\hline Midazolam & Dormicum & 7,5 \\
\hline Nitrazepam & Mogadan & 5 \\
\hline Nordazepam & Tranxilium N & 20 \\
\hline Oxazepam & Adumbran & 30 \\
\hline Prazepam & Demetrin & 20 \\
\hline Temazepam & Planum & 20 \\
\hline Tetrazepam & Musaril & 50 \\
\hline Triazolam & Halcion & 0,5 \\
\hline Zolpidem & Stilnox & 20 \\
\hline Zopiclon & Ximovan & 15 \\
\hline
\end{tabular}

1 Die angegebene Menge entspricht in ihrer Wirkung $10 \mathrm{mg}$ Diazepam bzw. $30 \mathrm{mg}$ Oxazepam.

Von Autor zu Autor finden sich für einzelne Präparate erhebliche Unterschiede in den Äquivalenzangaben, sodass diese Werte nur als Orientierung dienen sollten und im Einzelfall der klinischen Symptomatik angepasst werden müssen (s. unten).

\section{Halbwertszeit}

Trotz vergleichbarer Dosierung der BZD unterscheiden sich bei einer längerfristigen Anwendung die BZD wesentlich durch ihre verschiedenen Halbwertszeiten (s. Tab. 3).

Zum Teil werden die BZD in ebenfalls aktive BZD abgebaut, sodass sich deren Wirkdauer verlängert. Die Verstoffwechselung erfolgt gemäß einer Kinetik erster Ordnung - d. h., je mehr BZD im Körper vorhanden sind, umso mehr werden diese auch wieder ausgeschieden. Dadurch wird nach etwa der zweifachen Halbwertszeit ein steady-state-Zustand erreicht, bei dem zugeführte und ausgeschiedene Menge in etwa im Gleichgewicht stehen und die Menge des BZD nicht weiter kumuliert. Dies ist in Abb.1 optisch dargestellt für Diazepam und in Tab. 3 sind die verschiedenen Halbwertszeiten und Metabolite der BZD aufgelistet.

Durch die Kumulation ist die tatsächlich im Körper wirksame Menge an BZD weitaus höher, als die täglich eingenommene Menge. Bei dem Beispiel aus Abb.1 - tägliche Einnahme von $20 \mathrm{mg}$ Diazepam - bedeutet dies, dass sich die Menge an Diazepam im Körper auf $150 \mathrm{mg}$ erhöht hat (vereinfachte Rechnung unter der Vorgabe, dass Diazepam inklusive seiner aktiven Metabolite 150
Stunden Halbwertszeit besitzt - dies ist in Realität zwar länger, dafür sind die Metabolite schwächer wirksam).

Je nach Halbwertszeit des verwendeten BZD setzen die Entzugserscheinungen sehr rasch oder auch erst nach Tagen ein. Die Halbwertszeit spielt auch bei der Wahl des BZD eine Rolle, mit dem ausgeschlichen werden soll. Ist die Halbwertszeit zu kurz, treten zwischen den einzelnen Gaben des BZD relevante Plasmaspiegelschwankungen auf, die vom Patienten als Entzug empfunden werden. Ist die Halbwertszeit zu lang, tritt ggf. eine Kumulation auf und statt zu entziehen steigt beim Patienten zunächst die Plasmaspiegelkonzentration an (vgl. Abb.1). Dementsprechend sind Substanzen ohne aktiven Metaboliten und einer Halbwertszeit zwischen 15 und 25 Stunden als günstig anzusehen. Darüber hinaus muss das Präparat in verschiedenen Wirkstärken angeboten werden, damit die Tagesdosis möglichst auf vier Einzelgaben verteilt werden kann und in kleinen Schritten herunterdosiert werden kann.

Mit der Halbwertszeit lässt sich auch die Eliminationszeit errechnen, also die Zeit, bis eine Restmenge $X$ erreicht ist (da es sich um eine logarithmische Funktion handelt, wird rechnerisch der Wert Null nicht erreicht):

Anzahl Halbwertszeiten $=\log _{2}(1 /$ Restmenge $\times$ kumulierte Dosen $)$

Eliminationszeit $=\mathrm{HWZ} \times$ Anzahl $\mathrm{HWZ}$

z. B. bei Diazepam $1 \times \operatorname{tgl} .20 \mathrm{mg}$ nach 14 Tagen, HWZ $150 \mathrm{~h}$ (Anmerkung zur Halbwertzeit s. o.)

$\log _{2}(2 \times 150 \mathrm{mg})=8,2288$ Halbwertszeiten

*vorgegebene Restmenge $0,5 \mathrm{mg}$

Eliminationszeit $8,2288 \times 150 \mathrm{~h} \approx 1234 \mathrm{~h} \approx 51$ Tage

Diese errechnete Zeit ist länger, als sich BZD im Urin nachweisen lassen, da die Urintests im unteren Dosisbereich nicht mehr ausreichend sensibel messen (Vermeidung falsch positiver Ergebnisse durch Kreuzreaktionen).

\section{Entzugserscheinungen}

Das Symptommuster des Benzodiazepinentzuges ist vielfältig und nur schwer kalkulierbar und unterscheidet sich dadurch wesentlich vom Alkoholentzug. Bei einem Alkoholentzug lässt sich aufgrund aktueller Trinkmenge, Dauer der Trinkphase, lebenszeitlicher Trinkmenge und derzeitigen Allgemeinzustand die Schwere und Dauer des Entzuges relativ gut einschätzen. Die Symptome sind allgemein bekannt und lassen sich gut von Außen beobachten und durch Messung objektivieren. Nicht so beim Benzodiazepinentzug! Hier können Patienten nach 20 Jahren kontinuierlicher Einnahme, zuletzt im Hochdosisbereich, ohne nennenswerte Beschwerden das Präparat ausschleichen und andere, mit nur kurzer Einnahmedauer im Niedrigdosisbereich entwickeln ein merkliches Entzugssyndrom. Die dabei auftretenden Beschwerden sind sehr vielfältig und nicht spezifisch. Kompliziert wird dies durch das Problem, dass Symptome der Störung, die ursprünglich 


\begin{tabular}{|c|c|c|c|c|}
\hline Substanz & $\begin{array}{l}\text { Beispiele für } \\
\text { Handelsnamen }\end{array}$ & $\begin{array}{l}H W Z \\
\text { (in h) }\end{array}$ & Metabolit(en) & $\begin{array}{l}H W Z \\
\text { (in h) }\end{array}$ \\
\hline Alprazolam & Tafil $^{\circledR}$ & $10-15$ & Hydroxyalprazolam & $12-15$ \\
\hline Bromazepam & Lexotanil $^{\circledR}$, Normoc ${ }^{\circledR}$ & $10-20$ & Hydroxybromazepam & kurz \\
\hline Brotizolam & Lendormin ${ }^{\circledR}$ & $3-8,5$ & Hydroxymethyl-brotizolam & $3-8$ \\
\hline Chlodiazepoxid & Librium $^{\circledR}$ & $5-30$ & $\begin{array}{l}\text { Demoxepam } \\
\text { Nordazepam } \\
\text { Oxazepam }\end{array}$ & $\begin{array}{l}\text { ca. } 45 \\
36-200 \\
10-20\end{array}$ \\
\hline Clobazam & Frisium ${ }^{\circledR}$ & $12-60$ & Desmethyl-clobazam & $50-100$ \\
\hline Clonazepam & Rivotril $^{\circledR}$ & $30-40$ & $\begin{array}{l}\text { Aminoclonazepam } \\
\text { Acetaminoclonazepam }\end{array}$ & wirksam? \\
\hline Clotiazepam & Trecalmo $^{\circledR}$ & $3-15$ & $\begin{array}{l}\text { Desmethyl-clotiazepam } \\
\text { Hydroxy-clotiazepam }\end{array}$ & ca. 18 \\
\hline Diazepam & Valium $^{\circledR}$ & $20-40$ & $\begin{array}{l}\text { Nordazepam } \\
\text { Oxazepam }\end{array}$ & $\begin{array}{l}36-200 \\
10-20\end{array}$ \\
\hline Dikaliumclorazepat & Tranxilium ${ }^{\circledR}$ & $(1-2)$ & $\begin{array}{l}\text { Nordazepam } \\
\text { Oxazepam }\end{array}$ & $\begin{array}{l}36-200 \\
10-20\end{array}$ \\
\hline Flunitrazepam & Rohypnol $^{\circledR}$ & $16-35$ & $\begin{array}{l}\text { Aminoflunitrazepam } \\
\text { Desmethylflunitrazepam }\end{array}$ & wirksam? \\
\hline Flurazepam & $\begin{array}{l}\text { Dalmadorm }{ }^{\circledR}, \\
\text { Staurodorm neu }\end{array}$ & $40-250$ & - & \\
\hline Loprazolam & Sonin $^{\circledR}$ & $6-12$ & - & \\
\hline Lorazepam & Tavor $^{\circledR}$, Pro Dorm ${ }^{\circledR}$ & $8-24$ & - & - \\
\hline Lormetazepam & Noctamid $^{\circledR}$, Ergocalm ${ }^{\circledR}$ & $8-15$ & $\begin{array}{l}\text { (nur teilweise Abbau zu) } \\
\text { Lorazepam }\end{array}$ & $8-24$ \\
\hline Medazepam & Rudotel $^{\circledR}$, Rusedal ${ }^{\circledR}$ & & - & \\
\hline Metaclazepam & Talis $^{\circledR}$ & $7-23$ & Desmethyl-metaclazepam & $10-35$ \\
\hline Midazolam & Dormicum $^{\circledR}$ & 2 & & \\
\hline Nitrazepam & Mogadan $^{\circledR}$, Radedorm ${ }^{\circledR}$ & $15-38$ & - & \\
\hline Nordazepam & Tranxilium N ${ }^{\circledR}$ & $36-200$ & Oxazepam & $10-20$ \\
\hline Oxazepam & Adumbran $^{\circledR}$, Praxiten ${ }^{\circledR}$ & $10-20$ & - & - \\
\hline Prazepam & Demetrin $^{\circledR}$ & 1,5 & $\begin{array}{l}\text { Nordazepam } \\
\text { Oxazepam }\end{array}$ & $\begin{array}{l}36-200 \\
4-15\end{array}$ \\
\hline Temazepam & Musaril $^{\circledR}$ & $18-22$ & - & \\
\hline Triazolam & Halcion $^{\circledR}$ & 2,5 & - & - \\
\hline Zaleplon & Sonata $^{\circledR}$ & 1 & - & - \\
\hline Zolpidem & Bikalm $^{\circledR}$, Stilnox ${ }^{\circledR}$ & $1-3$ & - & - \\
\hline Zopiclon & Ximovan $^{\circledR}$ & $4-6$ & - & - \\
\hline
\end{tabular}

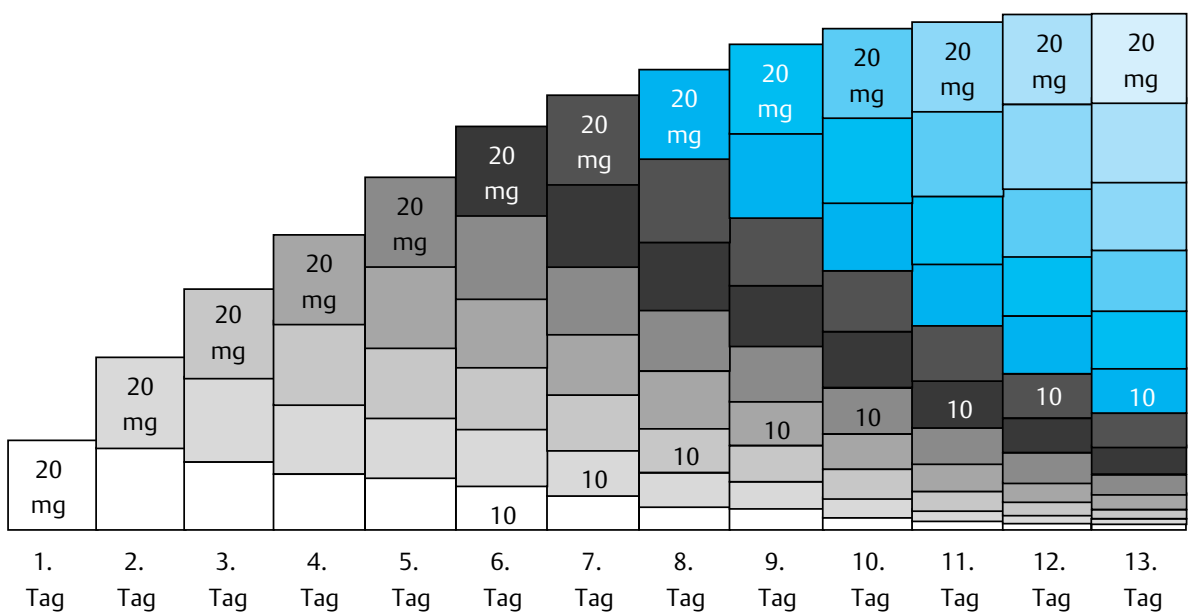

Abb. 1 Kumulation von Diazepam (unter der vereinfachten Annahme Halbwertszeit 150 Stunden [s. Text]).

zur Einnahme geführt haben, wieder auftreten können bzw. getriggert werden (z.B. Ängste). Besonders quälend können die Stimmungsschwankungen (mehrmals am Tag wechselnd), die intensivere Wahrnehmung („Dünnhäutigkeit“) und die qualitativ veränderte Wahrnehmung des eigenen Körpers bzw. der Körper- bewegungen sein. Tab. 4 gibt einen Überblick zu den häufigsten Entzugssymptomen.

Gerade die qualitativ veränderte (Körper-)Wahrnehmung gibt häufig Anlass zur neurologischen Differenzialdiagnostik. Keines- 
Tab. 4 Benzodiazepinentzugssymptome

\begin{tabular}{|c|c|}
\hline Unspezifische Symptome & Häufigkeit in \% \\
\hline Schlafstörungen & 71 \\
\hline Angst & 56 \\
\hline Verstimmung/Stimmungsschwankungen & 49 \\
\hline Muskelschmerzen/-zuckungen & 49 \\
\hline zittern & 38 \\
\hline Kopfschmerzen & 38 \\
\hline Übelkeit/Brechreiz/Appetitverlust & 36 \\
\hline schwitzen & 22 \\
\hline verschwommenes Sehen & 20 \\
\hline \multicolumn{2}{|l|}{ Wahrnehmungsstörungen } \\
\hline \multicolumn{2}{|l|}{ Überempfindlichkeit } \\
\hline + gegen Geräusche & 38 \\
\hline + gegen Licht & 24 \\
\hline + gegen Geruch & 15 \\
\hline + gegen Berührung & 7 \\
\hline \multicolumn{2}{|l|}{ Unterempfindlichkeit } \\
\hline - gegen Geruchsreize & 15 \\
\hline - gegen Geschmacksreize & 4 \\
\hline \multicolumn{2}{|l|}{ Qualitative Veränderung } \\
\hline Bewegungen & $>24$ \\
\hline Sehen & $>13$ \\
\hline Geschmack & 13 \\
\hline Hören & 2 \\
\hline Geruch & 2 \\
\hline \multicolumn{2}{|l|}{ Sonstige } \\
\hline Unwirklichkeitsgefühl & 24 \\
\hline \multicolumn{2}{|l|}{ Komplikationen } \\
\hline Psychosen & 7 \\
\hline epileptische Anfälle & 4 \\
\hline
\end{tabular}

nach $[1,13]$

falls sollten entsprechende Beschwerden automatisch als entzugsbedingt eingestuft werden, bevor nicht zumindest ein Minimum an entsprechender Diagnostik (z.B. körperlich neurologische Untersuchung) erfolgt ist.

\section{Patientenführung}

Die Behandlung von Medikamentenabhängigen im Entzug bedarf nicht nur des pharmakologischen Wissens, sondern auch einer fundierten psychiatrisch-psychotherapeutischen Kompetenz. Dies gilt umso mehr, je ausgeprägter eine ggf. zu Grunde liegende psychische Erkrankung ist.

Nachfolgend sind einige Empfehlungen zusammengefasst, die unabhängig vom Ausmaß der Komorbidität als „Basistherapie“ für Entzugspatienten hilfreich sind. Falls Patienten z.B. wegen einfachen Schlafstörungen oder einer vorübergehenden Krisensituation mit den Benzodiazepinen begonnen haben, so werden diese Empfehlungen in der Regel ausreichen, um die Betroffenen möglichst gut zu unterstützen.

Der Grundansatz dabei lautet: Die Betroffenen müssen wissen, warum sie mit der Einnahme aufhören sollen und was sie im Entzug erwartet.
Dazu muss den Patienten erklärt werden, weshalb „ihr“ Medikament nicht länger eingenommen werden soll. Vielfach besteht schon eine „Ahnung“ davon, dass das entsprechende Präparat nicht längere Zeit genommen werden soll und abhängig macht. Dieses Wissen schreckt aber genauso wenig ab wie der Aufdruck auf den Zigarettenpackungen („Rauchen kann tödlich sein“). Die Betroffenen müssen die konkreten, im Alltag bemerkbaren Symptome genannt bekommen und überprüfen, inwieweit sie schon diese Veränderungen bei sich bemerkt haben. Dazu kann die Tab. 1 herangezogen werden. Diese Informationen müssen ergänzt werden mit dem Hinweis auf Wirkverlust und Wirkumkehr (s.o.). Interessant ist das Phänomen, dass gerade Patienten im Niedrigdosisbereich im Verlauf auch unter der Einnahme Entzugserscheinungen aufweisen. Ähnlich wie bei Alkoholikern, die in der fortgeschrittenen Phase ihrer Erkrankung immer höhere Blutalkoholkonzentrationen benötigen, ist es auch bei den BZD. Wird dieser Spiegel nicht erreicht, stellen sich Entzugserscheinungen ein, obwohl - wie beim Beispiel des Alkoholikers noch Restalkohol im Körper ist. Dieses Phänomen erklärt vermutlich auch die Wirkumkehr, da Entzugserscheinungen im Wesentlichen die entgegengesetzten Effekte der Substanzwirkungen sind (beim Beispiel Alkohol die schlafanstoßende Wirkung des Alkohols und im Entzug dann Unruhe und Schlaflosigkeit).

Die verschiedenen Symptome, die in Tab. 1 genannt sind, sind unspezifischer Art. Auch hier lässt sich unter Umständen die Angst, die zum Beginn der Einnahme führte nicht von der Angst im Sinne einer Wirkumkehr sicher unterscheiden, auch wenn der Verlauf der Beschwerden einen Hinweis geben kann (Angst unter BZD zunächst besser, im Verlauf der Einnahme erneute Zunahme der Ängste ohne dass spezifische Ursachen im Lebensumfeld dies erklären könnten). Diese Unsicherheit sollte immer benannt werden. Nur ein schrittweises Absetzen wird eine Antwort bringen. Gerade Patienten mit einer Wirkumkehr profitieren sehr rasch, unter Umständen schon in der Phase der Reduktion, vom Entzug. Unter Umständen kann es hilfreich sein, Angehörige oder Freunde mit in eines solches Aufklärungsgespräch hineinzunehmen, da vielfach die Betroffenen die sich über Monate und Jahre entwickelnden Veränderung nicht so deutlich wahrnehmen.

Je nach Halbwertszeit des verwendeten Präparates, sollten die Betroffenen auch über die Problematik der Kumulation (s.o. und Abb.1) aufgeklärt werden. Insbesondere ältere Menschen (und ihre Angehörigen) müssen um das Sturzrisiko unter BZD wissen (Dämpfung mit schlechterer Wahrnehmung und Reaktionsvermögen plus Muskelentspannung), dass bei BZD-Präparaten mit langer Halbwertszeit auch noch tagsüber besteht.

Solche Gespräche brauchen Zeit, die im Praxisalltag fehlt. Sicherlich nicht als Ersatz, aber doch als Vorbereitung oder Ergänzung können Informationsbroschüren dienen (z.B. von der DHS und dem BKK-Bundesverband herausgegebene Heft „Immer mit der Ruhe ...."). Die Erfahrung zeigt, dass fast alle Patienten sich, entsprechend informiert, für einen Entzug entscheiden.

Auch über den Entzug muss realistisch aufgeklärt werden. Als Informationsgrundlage kann dazu die Tab. 4 verwendet werden. Die Patienten können auch gut damit umgehen, dass im Vorfeld keine Abschätzung über die Schwere des Entzuges möglich ist. 
Entscheidend ist das Gefühl, jetzt eine angemessene Hilfe zu bekommen. Dementsprechend ist mit dem Betroffenen zu erörtern, welche Formen der Unterstützung zur Verfügung stehen. Offen bleibt zum Zeitpunkt vor dem Entzug, ob danach eine Behandlung z.B. der Schlafstörungen überhaupt noch notwendig ist. Für die Patienten ist es jedoch wichtig zu wissen, dass sie nach dem Entzug eine angemessene Hilfe bekommen können und wie diese aussieht.

Ein großes Thema bei Medikamentenabhängigen sind Schlafstörungen. Sie werden besonders gefürchtet und sind bei vielen Patienten der Einstieg in die BZD-Verschreibung gewesen. Patienten sollten deshalb parallel zur Vorbereitung bzw. spätestens zu Beginn des Entzuges zur Schlafhygiene beraten werden.

\section{Tipps für einen besseren Schlaf}

- Nicht tagsüber Schlafen,

- Schlafzimmer kühl und dunkel,

- keine körperlichen Anstrengungen vor dem Schlafengehen,

- keine aufputschenden Getränke abends - eventuell bereits nachmittags weglassen,

- keine schweren Mahlzeiten am Abend,

- wenn möglich „Bettschwere“ abwarten,

- Zu-Bett-Geh-Ritual,

- Bett ausschließlich zum Schlafen aufsuchen,

- feste Zeiten - insbesondere feste morgendliche Aufstehzeit,

- Wecker/Uhren aus Sichtweite,

- wenn wieder wach: Liegen bleiben und sich sagen: „Der Körper holt sich den Schlaf den er braucht - auch nur so zu liegen erholt mich ....",

- wenn Aufstehen: Licht auslassen.

Wer näheres dazu erfahren möchte, kann sich unter http://www.lwl.org/LWL/Gesundheit/psychiatrieverbund/K/ klinik_warstein/leistung/sucht/weiterfuehrende_

fachinformationen/ weiterführende Erläuterungen herunterladen.

Auch Ängste sind ein häufiges Thema. Bei schweren Ängsten wird in der Regel ein stationärer Entzug unvermeidbar sein, um bei vermehrten Ängsten oder Panikattacken den Betroffenen ohne BZD unterstützen zu können. Ideal ist, wenn bereits auf der Entzugsstation mit einer angstspezifischen Therapie begonnen werden kann oder zumindest im Sinne einer Angst/Informationsgruppe erste Hilfestellungen und weitere Behandlungsperspektiven aufgezeigt werden.

Auch klinisch relevante depressive Syndrome sollten zeitnah zum Entzug behandelt werden. Bei einer harten Indikation für ein Antidepressivum (z.B. klarer phasenhafter Verlauf der Depression) kann parallel zum Entzug mit einer entsprechenden Therapie begonnen werden, wobei die krampfschwellensenkende Wirkung der meisten Antidepressiva berücksichtigt werden sollte. Ist die Anamnese nicht eindeutig, sollte bis nach dem Entzug gewartet werden, ob sich ein depressives Syndrom ausbildet bzw. verstärkt. Besteht bereits vor dem Entzug ein mindestens mittelschweres depressives Syndrom, ist ebenfalls bereits vor bzw. parallel zum Entzug mit einem Antidepressivum zu beginnen, um den Patienten den Entzug zu erleichtern.
Soll der Entzug ambulant erfolgen, muss geprüft werden, ob die aktuelle Lebenssituation und Lebensführung einen Entzug zulässt. Lebt z. B. ein Betroffener ohne jegliche Tagesstruktur und Ziel und setzt die BZD ein, um möglichst viel zu schlafen, so muss zunächst wieder ein normaler Tag/Nachtrhythmus und eine Tagesstruktur etabliert werden.

Eine Gruppe anderer Betroffener, die aktuell ebenfalls einen BZD-Entzug absolvieren, wird von den allermeisten Patienten als extrem hilfreich erlebt, da der Austausch über die Entzugssymptome und weitere psychische Beschwerden die Patienten aus der Isolation mit den Tabletten herausholt und der Verzicht auf die Tabletten durch die positiven Erfahrungen von anderen Gruppenmitgliedern, die schon weiter sind, wesentlich leichter fällt [10].

Im nächsten Kapitel werden die pharmakologischen Strategien im BZD-Entzug erläutert. Natürlich müssen die Patienten auch darüber aufgeklärt sein und im Vorfeld des Entzuges entsprechend instruiert werden. Durch die zumeist passive Grundhaltung der Medikamentenabhängigen, verbunden mit der Tendenz die Verantwortung für die eigene (seelische) Gesundheit an den Arzt zu delegieren, besteht mit jeder Medikamentengabe bei dieser Patientengruppe die Gefahr, dieses Muster zu bedienen. Die Kognition der Patienten dabei ist, dass das „böse“ gegen ein „gutes“ Medikament ausgetauscht wird, weil sie jetzt endlich einen „guten Arzt“ gefunden haben, der weiß, wie sie behandelt werden müssen. Dieser Gedanke greift aber auf jeden Fall zu kurz und es ist wichtig, den Patienten deutlich zu machen, dass die Entzugsmedikamente (oder Antidepressiva gegen Depression, Angst, Schlafstörung) nur ein Teil der Behandlung sind. Viel wichtiger ist der Anteil, den der Patient mit einbringen muss, z.B. durch die Bereitschaft neue (Entzugs-)Beschwerden auszuhalten, eine Psychotherapie zu beginnen, sein Leben zu verändern.

\section{Konkrete pharmakologische Vorgehensweise}

Früher wurden Benzodiazepinentzüge häufig wie Alkoholentzüge durch schlagartiges Absetzen eingeleitet. Diese Methode ist obsolet, da das Risiko für ein Entzugsdelir bzw. einen epileptischen Anfall dadurch sehr hoch ist. Auch kommt die Mehrzahl der Patienten mit dem schlagartigen Absetzen „ihres“ Medikamentes aus psychologischen Gründen nicht zurecht und die im Vergleich zum schrittweisen Abdosieren wesentlich heftigeren Entzugserscheinungen sind für eine große Zahl an Patienten schlicht unerträglich.

Es gibt eine Vielzahl an Methoden, wie das Abdosieren erfolgen soll. Es fehlt an entsprechenden Studien, sodass auch die Leitlinie zur Behandlung der Medikamentenabhängigkeit keine Methode eindeutig favorisiert [9]. Eindeutig ist allerdings, dass der Entzug mit einem ausreichend lang wirksamen Benzodiazepin durchzuführen ist, da dies die Plasmaspiegelschwankungen über den Tag vermindert und so die Abbruchquote und die frühe Rückfallrate senkt [15-16].

Als erste beschrieben Klein et al. [17] den Benzodiazepinentzug mit Carbamazepin. 
Mittlerweile liegen einige Studien vor, bei denen unter Carbamazepin das Benzodiazepin z.T. schlagartig abgesetzt wurde [18-21]. Allen Studien ist gemeinsam, dass Carbamazepin die Entzugserscheinungen senkte (überwiegend signifikant), ohne dass wesentliche Nebenwirkungen auftraten.

In den Bereich der „suchtmedizinischen Glaubensfragen“ gehört die Diskussion um verdeckten versus offenen Entzug. Beim „verdeckte Entzug“ erfährt der Patient nicht seine aktuelle Dosierung und damit die Reduktionsschritte, um die Erwartungsangst zu reduzieren, die viele Betroffene bei jedem „Viertelchen weniger“ haben. Dafür müssen die Patienten „blindes Vertrauen“ zum Arzt haben, dass dieser sie nicht falsch behandelt - das ist das Gegenteil von dem, was für Medikamentenabhängige zukünftig sinnvoll ist. Der Vorteil des „offenen Entzuges“ ist die Möglichkeit, Erwartungsangst und das „Feilschen um die Dosis“ therapeutisch zu nutzen und mit dem Patienten seine süchtigen und abhängigen Verhaltensweisen wahrzunehmen und zu bearbeiten. Bei ambulanten Entzügen wird es in der Regel keine Alternative zum „offenen Entzug“ geben, der sich auch zunehmend im stationären Bereich durchgesetzt hat.

Die Frage nach ambulantem oder stationärem Entzug hängt vor allem davon ab, wie ausführlich und engmaschig die Patienten ambulant gesehen werden können und was der Patient sich selber zutraut. Der ambulante Entzug beansprucht erheblich mehr Zeit, da die Reduktionsschritte nicht zu schnell aufeinander folgen sollten und meistens niedriger sind, als im stationären Rahmen.

Bei einer Hochdosisabhängigkeit (über $20 \mathrm{mg}$ Diazepamäquivalenzdosis, s. Tab. 2) wird zunächst (stationär ab Aufnahme, ambulant vor Umstellung der BZD [s.u.]) Carbamazepin in der üblichen therapeutische Dosierung von 600-1200 mg an Verträglichkeit und Plasmaspiegel orientiert $(4-10 \mu \mathrm{g} / \mathrm{ml})$ oder Valproinsäure (Ziel $50-100 \mu \mathrm{g} / \mathrm{ml}$ mit einer Dosis von $1200 \mathrm{mg}$ ) gegeben.

Dann erfolgt die Umstellung auf ein Benzodiazepin mit mittlerer Halbwertszeit. Es empfiehlt sich stets den gleichen Wirkstoff zu verwenden, um damit ausreichend Routine und Erfahrung zu entwickeln. Da Oxazepam, ein BZD mit mittlerer Halbwertszeit, im Handel sowohl als $10 \mathrm{mg}$ als auch als $50 \mathrm{mg}$ Tabletten angeboten wird, ist es auch von der Tablettenstückelung günstig - sowohl hohe BZD-Dosierungen werden abgedeckt als auch im niedrigen Dosisbereich sind kleine Reduktionsschritte möglich. Die bisherige Dosierung wird anhand Tab. 2 in eine entsprechende Oxazepamdosis umgerechnet. Im ambulanten Bereich wird die Dosis ähnlich verteilt, wie der Patient sie bisher eingenommen hat, im stationären Bereich sollte von Anfang an die Dosis auf vier Gaben verteilt werden, wobei der tageszeitliche Schwerpunkt entsprechend der bisherigen Einnahme des Patienten gewählt werden kann.

Uneinheitlich ist die Auffassung bezüglich einer Maximaldosis, die gegeben werden soll. Natürlich haben viele Patienten die Tendenz eine höhere (manche aber auch eine niedrigere) Dosis anzugeben, als sie tatsächlich genommen haben, unter der Vorstellung, mit einer erhöhten Einstiegsdosis besser durch den Entzug zu kommen. Einige Kliniken geben deshalb generell nicht mehr als 120 mg oder $180 \mathrm{mg}$ Oxazepam. Rein pharmakologisch ist bei einer Dosis über
$180 \mathrm{mg}$ Oxazepam auch kein weiterer Effekt zu erwarten (außer Intoxikation); in der Praxis zeigt sich aber, dass Patienten, die $300 \mathrm{mg}$ Oxazepamäquivalenzdosis und mehr konsumiert haben (gerade Patienten mit BZD-,Beikonsum“ bei Polytoxikomanie) mit höheren Dosierungen behandelt werden müssen. Es empfiehlt sich in diesen Fällen, die Dosisfindung trotzdem von einer niedrigen Dosierung (180 mg Oxazepam) aus zu beginnen und nicht gleich mit Dosierungen über $240 \mathrm{mg}$ einzusteigen.

Sehr selten sind Patienten, die über Nacht so deutliche Plasmaspiegelabfälle erleben, dass auf eine Substanz wie Clonazepam umgestiegen werden muss, die eine längere Halbwertszeit hat. Da es Clonazepam auch als Tropfen gibt, lässt es sich gut abdosieren. Es gibt allerdings Patienten im ambulanten Rahmen, die mit den Tropfen Schwierigkeiten entwickeln und zusätzliche Tropfen nehmen, was zunächst auch nicht auffällt.

Die Patienten müssen generell daraufhin befragt bzw. beobachtet werden, ob die Dosierungen richtig gewählt wurde. Beim ambulanten Entzug muss der Arzt zumindest telefonisch für den Patienten erreichbar sein, um bei Über- oder Unterdosierung Dosiskorrekturen absprechen zu können. Erschwerend kommt hinzu, dass je nach Halbwertszeit des verwendeten Präparates im Verlauf die Dosis noch einmal (nach oben) angepasst werden muss. Bei BZD mit kurzer Halbwertszeit sinkt der Spiegel des bisher genommenen BZD rasch ab und bei zu niedriger Dosierung von Oxazepam wird zeitnah reagiert werden müssen. Bei BZD mit sehr langer Halbwertszeit und hoher Dosierung werden die Patienten unter Umständen die ersten Tage unabhängig von der gewählten Oxazepamdosis noch Zeichen der Intoxikation aufweisen und erst nach der Dauer einer Halbwertszeit des bisher genommenen Präparates wird eine angemessene Dosis zu finden sein.

Im ambulanten Bereich wird nach einer Woche der nächste Schritt erfolgen und Oxazepam auf (mindestens) zwei Gaben verteilt (kontinuierlicherer Plasmaspiegelabfall bei mehrmals täglicher Gabe).

Stationär wird in den ersten zwei Tagen der Eingewöhnung die ambulante BZD-Dosis mit Oxazepam fortgeführt.

Im stationären Rahmen wird bei einer Hochdosisabhängigkeit nach folgendem Muster reduziert:

Bei Dosierung über 100 mg Oxazepam 50 mg Reduktionsschritte (täglich)

Ab 100 mg Oxazepam in 25 mg Schritten (alle 2 Tage)

Ab 50 mg Oxazepam Reduzierung in 10 mg Schritten (alle 2 Tage)

Ab 20 mg Oxazepam Reduzierung in 5 mg Schritten (alle 2 Tage)

Im stationären Rahmen wird bei einer Niedrigdosisabhängigkeit ( $\leq 60 \mathrm{mg}$ Oxazepam) nach folgendem Muster reduziert:

Bei einer Dosierung von $60 \mathrm{mg}$ Oxazepam erster Reduktionsschritt auf $50 \mathrm{mg}$, danach alle 2 Tage um $5 \mathrm{mg}$ Oxazepam reduzieren: 


\begin{tabular}{|rrrr|}
\hline $20-$ & $10-$ & $10-$ & 20 \\
\hline $10-$ & $10-$ & $10-$ & 20 \\
$10-$ & $5-$ & $10-$ & 20 \\
$10-$ & $5-$ & $5-$ & 20 \\
$10-$ & $5-$ & $5-$ & 15 \\
\hline $5-$ & $5-$ & $5-$ & 15 \\
\hline $5-$ & $0-$ & $5-$ & 15 \\
\hline $5-$ & $0-$ & $5-$ & 10 \\
\hline $5-$ & $0-$ & $0-$ & 10 \\
\hline $5-$ & $0-$ & $0-$ & 5 \\
\hline $0-$ & $0-$ & $0-$ & 5 \\
\hline
\end{tabular}

Besonders schwierig wird es für die Patienten bei den letzten Reduktionsschritten. Im Verlauf muss deshalb individuell die Reduktionsgeschwindigkeit reduziert werden und der nächste Reduktionsschritt einen Tag ausgesetzt werden.

Für ambulante Entzüge wird die Vorgehensweise modifiziert: Die Reduktionsschritte erfolgen wöchentlich. Die Reduktionsschritte können natürlich auch zeitlich gestreckt werden, dann ist aber zu bedenken, dass sich der Entzug sehr lange hinzieht und eine entsprechende Ausdauer von Nöten ist.

Bei starker Unruhe, quälender Schlafstörung oder deutlicher depressiver Symptomatik ist eine Zusatzmedikation mit einem sedierenden Antidepressivum wie Doxepin $(2 \times 25 \mathrm{mg}$ oder $50 \mathrm{mg}$ z. N.) oder Mirtazapin (15 oder $30 \mathrm{mg}$ ) angezeigt. Der Einsatz von Baldrianpräparaten hat sich in Studien im Hinblick auf Schlafstörungen ebenfalls bewährt [22 - 23]. Diese Art von Medikation muss aber mit dem Patienten gut besprochen werden, da durch die Selbstmedikationsmöglichkeit ggf. nur das BZD durch Baldrian ersetzt wird, ohne dass sich an der passiven Grundhaltung etwas verändert. Hier bestehen im suchtmedizinischen Kontext sicherlich andere Ansprüche, als bei der hausärztlichen Versorgung im Altenheim.

Carbamazepin bzw. Valproinsäure kann wieder ausgeschlichen werden, wenn im Urin keine BZD mehr nachweisbar sind und die Entzugssymptome abgeklungen sind. Bei Menschen mit erhöhtem Risiko für epileptische Anfälle (Schädel-Hirn-Trauma, positive Familienanamnese, eigene epileptische Anfälle) sollte vorher noch ein EEG abgeleitet werden.

Falls sich beim Abdosieren von Carbamazepin die Entzugssymptome verstärken sollten, so ist es im Einzelfall anzuraten, Carbamazepin bis zu einem Jahr weiter zu geben.

Es gibt keine klare zeitliche oder messtechnische Grenze für das Ende des Entzuges. Messungen der Benzodiazepine und ihrer Metabolite im Urin sind sehr störanfällig (Trinkmenge, sportliche Aktivität, erster Morgenurin oder später, ...). In der Regel brauchen die Patienten noch zwei bis vier weitere Wochen zur Stabilisierung, in denen sie unterstützt werden müssen zu lernen, mit der veränderten Wahrnehmung und Empfindung ohne BZD umzugehen und die beständig oder intermittierend auftretenden Entzugssymptome zu bewältigen.

Einige Patienten haben noch über Monate (Einzelfälle auch noch länger) - zumindest phasenweise - Entzugssymptome. Für diese
Patientengruppe ist eine weitere Betreuung sehr anzuraten und die Wiederherstellung der Arbeitsfähigkeit erst im weiteren Verlauf zu erwarten.

Falls gleichzeitig eine Abhängigkeit von Alkohol besteht so wird die Vorgehensweise modifiziert:

Wenn das Benzodiazepin zuletzt die führende Substanz war, wird mindestens die (realistische) letzte Dosis in äquivalenter Oxazepamdosis weiter gegeben (s.o.). Ggf. muss bei der Ausbildung von merklichen Alkohol-Entzugserscheinungen die Dosis im Verlauf angepasst werden. Die Reduktion von Oxazepam erfolgt nach Abklingen des Alkoholentzuges (ab ca. vierten Tag) wie oben beschrieben.

Wenn der Alkohol zuletzt die führende Substanz war, wird Oxazepam gemäß der Alkoholentzugssymptomatik gegeben und die Abdosierung erfolgt zügiger.

Nicht zu empfehlen ist die Kombination Benzodiazepin und Clomethiazol (Distraneurin ${ }^{\circledR}$ ), da beide sedierend und in höheren Dosen atemdepressiogen sind und sich so Nebenwirkungsrisiken unkalkulierbar summieren.

Versuche Entzugserscheinungen mit Betablockern, Buspiron, Clonidin oder Valproinsäure abzufangen, blieben ohne großen Erfolg [24-27].

Antidepressiva zur allgemeinen Milderung von Entzugssymptomen bei nicht depressiven Abhängigen bringt nur mäßigen Erfolg [28].

Flumazenil (partieller Benzodiazepinrezeptoragonist) wurde im Tierexperiment im Benzodiazepinentzug mit gutem Erfolg eingesetzt. Erste Studien beim Menschen liegen vor [29], wobei sich eine Empfehlung für den klinischen Alltag daraus noch nicht ableiten lässt.

Ebenfalls positive tierexperimentelle Befunde liegen zur Gabe von Calciumkanalblockern vor [30].

Mit Abschluss des Entzuges darf die Behandlung nicht aufhören. Selbsthilfegruppen, Nachsorgegruppen, Entwöhnungstherapie oder eine Einzeltherapie sind die Hauptoptionen. Pharmakologisch orientiert sich die Behandlung an den klinischen Symptomen bzw. der ggf. weiteren psychiatrischen Erkrankung. Eine spezifische Ersatz- oder Rückfallschutzsubstanz (Anti craving) gibt es nicht.

\section{Literatur}

${ }^{1}$ Laux G, König W. Long-term use of benzodiazepines in psychiatric inpatients. Acta psychiatr scand 1987; 76: $64-70$

2 Arolt V, Driessen M, Dilling H. Psychische Störungen bei Patienten im Allgemeinkrankenhaus. Dt Ärzteblatt 1997; 94: A 1354-1358

${ }^{3}$ Lemoine P, Touchon J, Billardon M. Comparison o 6 different methods for lorazepam withdrawal. A controlled study, hydroxyzyne versus placebo. Encephale 1997; 23: 290-299

${ }^{4}$ Pollack MH, Allgulander C, Bandelow B et al. World Council of Anxiety: WCA recommendations for the long-term treatment of panic disorder. CNS Spectr 2003; 8 (Suppl 1): 17-30 
${ }^{5}$ Allgulander C, Bandelow B, Hollander E et al. World Council of Anxiety. WCA recommendations for the long-term treatment of generalized anxiety disorder. CNS Spectr 2003; 8 (Suppl 1): 53-61

${ }^{6}$ Holbrook AM, Crowther R, Lotter A et al. Meta-analysis of benzodiazepine use in the treatment of acute alcohol withdrawal. Can Med Ass J 1999; 160: 649-655

${ }^{7}$ Nowell PD, Mazumdar S, Buysse DJ et al. Benzodiazepines and zolpidem for chronic insomnia: a meta-analysis of treatment efficacy. JAMA 1997; 278 (24): $2170-2177$

${ }^{8}$ Agency for Healthcare Research and Quality. www.ahcpr.gov

${ }^{9}$ Poser W, Böning J, Holzbach R et al. Medikamentenabhängigkeit In: Schmidt LG, Gastpar M, Falkai P, Gaebel W (Hrsg). Evidenzbasierte Suchtmedizin. Köln: Deutscher Ärzteverlag, 2006: 271 - 308

${ }^{10}$ Holzbach R. Weiter verschreiben oder stoppen? Die Langzeiteinnahme von Benzodiazepinen. Der Neurologe \& Psychiater 2005; 10: 45 - 47

${ }^{11}$ Schweizer E, Case WG, Rickels K. Benzodiazepine dependence and withdrawal in elderly patients. Am J Psychiatry 1989; 146: 529-531

${ }^{12}$ Sternbach LH. The Benzodiazepine Story. Journal of Psychoactive Drugs 1983; $15(1-2): 15-17$

${ }^{13}$ Schöpf J. Withdrawal phenomena after long term administration of benzodiazepines. A review of recent investigations. Pharmacopsychiatry $1983 ; 16: 1-8$

${ }^{14}$ Laux G. Aktueller Stand der Behandlung mit Benzodiazepinen. Nervenarzt 1995; 66: $311-322$

${ }^{15}$ Murphy S, Tyrer P. A Double-Blind Comparison of the Effects of Gradual Withdrawal of Lorazepam, Diazepam and Bromazepam in Benzodiazepine Dependence. British Journal of Psychiatry 1991; 158: $511-516$

${ }^{16}$ Rickels K, Case WG, Schweizer E et al. Benzodiazepine Dependence. Management of Discontinuation. Psychopharm Bull 1990; 26 (1): 63-68

${ }^{17}$ Klein E, Uhde TW, Post RM. Preliminary evidence for the utility of carbamazepine in alprazolam withdrawal. Am J Psychiatry 1986; 143: $235-236$

18 Ries RK, Roy-Byrne PP, Ward NG et al. Carbamazepine treatment for benzodiazepine witdrawal. Am J Psychiatry 1989; 146: 536-537
${ }^{19}$ Schweizer E, Rickels K, Case WG et al. Carbamazepine treatment in patients discontinuing long term benzodiazepine therapy. Arch Gen Psychiatry 1991; $48: 448-452$

${ }^{20}$ Di Costanzo E, Rovea A. The prophylaxis of benzodiazepine withdrawal syndrome in the elderly: the effectiveness of carbamazepine. Double-blind study vs. placebo. Minerva Psychiatrica 1992; 33 (4): $301-304$

${ }^{21}$ Kaendler SH, Volk S, Pflug B. Benzodiazepinentzug mit Carbamazepin. Nervenarzt 1996; 67: $381-386$

${ }^{22}$ Poyares D, Guillemninault C, Ohayon M et al. Chronic benzodiazepine usage and withdrawal in insomnia patients. J Psychiatr Res 2004; 38: $327-334$

${ }^{23}$ Poyares D, Guillemninault C, Ohayon M et al. Can valerian improve the sleep of insomniacs after benzodiazepine withdrawal? Prog Neuropsychopharmacol Biol Psych 2002; 26: 539-545

24 Tyrer P, Rutherford D, Hugget T. Benzodiazepine withdrawal symptoms and propanolol. Lancet 1981; I: 520-522

${ }^{25}$ Schweizer E, Rickels K. Failure of buspirone to manage benzodiazepine withdrawal. Am J Psychiatry 1986; 143: 1590-1592

${ }^{26}$ Vinogradow S, Reiss AL, Cernansky JG. Clonidine therapy in withdrawal from high-dose alprazolam treatment. Am J Psychiatry 1986; 143: 1188

27 Apelt S, Emrich HM. Sodium valproate in benzodiazepine withdrawal. Am J Psychiatry 1990; 147: 950 - 951

28 Tyrer P, Ferguson B, Hallstrom C et al. A controlled trial of dothiepin and placebo in treating benzodiazepine withdrawal symptoms. British Journal of Psychiatry 1996; 168: 457-461

${ }^{29}$ Saxon L, Hjemdahl P, Hiltunen AJ et al. Effects of flumazenil in the treatment of benzodiazepine withdrawal - a double-blind pilot study. Psychopharmacology 1997; 131: $153-160$

${ }^{30}$ Gupta MB, Nath C, Patnaik GK et al. Effect of calcium channel blockers on withdrawal syndrome of lorazepam in rats. Indian Journal of Medical Research 1996; 103: 310-314 